TITLE:

\title{
Quantum effects of hydrogen atoms on the dynamical rearrangement of hydrogen-bond networks in liquid water.
}

\section{$\operatorname{AUTHOR}(S)$ :}

Kim, Hyeon-Deuk; Ando, Koji

\section{CITATION:}

Kim, Hyeon-Deuk ...[et al]. Quantum effects of hydrogen atoms on the dynamical rearrangement of hydrogen-bond networks in liquid water.. The Journal of chemical physics 2010, 132(16): 164507.

\section{ISSUE DATE:}

2010-04-28

URL:

http://hdl.handle.net/2433/120796

RIGHT:

(C) 2010 American Institute of Physics 


\title{
Quantum effects of hydrogen atoms on the dynamical rearrangement of hydrogen-bond networks in liquid water
}

\author{
Kim Hyeon-Deuk ${ }^{\text {a) }}$ and Koji Ando \\ Department of Chemistry, Kyoto University, Kyoto 606-8502, Japan
}

(Received 17 January 2010; accepted 29 March 2010; published online 26 April 2010)

\begin{abstract}
Quantum effects such as zero-point energy and delocalization of wave packets (WPs) representing water hydrogen atoms are essential to understand anomalous energetics and dynamics in water. Since quantum calculations of many-body dynamics are highly complicated, no one has yet directly viewed the quantum WP dynamics of hydrogen atoms in liquid water. Our semiquantum molecular dynamics simulation made it possible to observe the hydrogen WP dynamics in liquid water. We demonstrate that the microscopic WP dynamics are closely correlated with and actually play key roles in the dynamical rearrangement in the hydrogen-bond network (HBN) of bulk water. We found the quantum effects of hydrogen atoms on liquid water dynamics such as the rearrangement of HBN and the concomitant fluctuation and relaxation. Our results provide new physical insights on HBN dynamics in water whose significance is not limited to pure liquid dynamics but also a greater understanding of chemical and biological reactions in liquid water. () 2010 American Institute of Physics. [doi:10.1063/1.3397809]
\end{abstract}

\section{INTRODUCTION}

Chemical reactions taking place in water are essential for many important processes in electrochemical, environmental, pharmaceutical, and biological sciences. The unique and anomalous properties of water stem from its dynamics of hydrogen-bond networks (HBNs). Many attempts have been conducted to clarify how liquid water is dynamically organized via the HBNs. Computer simulation has been a strategy of particular importance in this field. A number of empirical potentials have been developed for better agreement with experiments using the parameters calibrated for use in full classical water (FCW) molecular dynamics (MD) and in Monte Carlo simulations. ${ }^{1-8}$ The classical MD is nowadays very straightforward and is routinely used to study complex chemical problems involving many thousands of particles. ${ }^{3,9}$ In contrast, quantum-mechanical simulations are not yet established for many-body dynamics in condensed phases, despite the fact that quantum effects such as zero-point energy (ZPE), delocalization of WPs describing water hydrogen atoms, etc., are envisaged to be essential in the HBN dynamics. ${ }^{10,11}$ Although considerable progress has been made in the development of exact quantum-mechanical methods, they are yet applicable to relatively small systems and limited times and provide only the static spacial and momentum distributions. ${ }^{12,13}$ In order to capture such quantum effects in many degrees of freedom, a number of approaches exploiting semiquantum and mixed quantumclassical simulations have been proposed and applied in recent years. The path-integral Monte Carlo (PIMC) method was used to assess the effect of quantum-mechanical fluctuations on the liquid structure, ${ }^{14-18}$ and the centroid MD (CMD), ring polymer MD, and semiclassical initial value

\footnotetext{
${ }^{a)}$ Author to whom correspondence should be addressed. Electronic mail: kim@kuchem.kyoto-u.ac.jp.
}

representation methods were proposed to study the water dynamics through equilibrium correlation functions and meansquare displacement. ${ }^{19-29}$ However, no one has directly viewed the quantum dynamics of water hydrogen atoms in condensed bulk water and their effects on the rearrangement of HBN and the concomitant fluctuation and relaxation. ${ }^{1,30} \mathrm{In}$ this study, we report a direct simulation study of hydrogen WP dynamics in liquid water and suggest its key role in the dynamical rearrangement of the HBN.

\section{THEORY AND SIMULATION DETAILS}

In order to take account of the nuclear quantum aspects such as the WP delocalizing and the ZPE effects in the liquid water dynamics, the hydrogen atoms are described by threedimensional (3D) $N$-independent symmetric Gaussian WP basis functions of a squeezed coherent state $\Psi\left(\left\{x_{i}, y_{i}, z_{i}\right\}, t\right)$ $=\prod_{i=1}^{N} \Psi_{i}\left(x_{i}, y_{i}, z_{i}, t\right)$, where

$$
\begin{aligned}
\Psi_{i}\left(x_{i}, y_{i}, z_{i}, t\right)= & N_{i} \exp \left[A _ { i } ( t ) \left\{\left(x_{i}-q_{x_{i}}(t)\right)^{2}+\left(y_{i}-q_{y_{i}}(t)\right)^{2}\right.\right. \\
& \left.+\left(z_{i}-q_{z_{i}}(t)\right)^{2}\right\}+i p_{x_{i}}(t)\left\{x_{i}-q_{x_{i}}(t)\right\} \\
& \left.+i p_{y_{i}}(t)\left\{y_{i}-q_{y_{i}}(t)\right\}+i p_{z_{i}}(t)\left\{z_{i}-q_{z_{i}}(t)\right\}\right] .
\end{aligned}
$$

$q_{\alpha_{i}}(t)$ and $p_{\alpha_{i}}(t)$ with $\alpha_{i}=x_{i}, y_{i}$ or $z_{i}$ are the 3D Cartesian coordinates of the WP center and their momenta, respectively. (For simplicity, $\hbar=1$ and all the coordinates are mass scaled.) $A_{i}$ is defined by $A_{i}(t)=\left(-1+2 i \rho_{i}(t) \Lambda_{i}(t)\right) / 4 \rho_{i}^{2}(t)$, where $\rho_{i}(t)$ and $\Lambda_{i}(t)$ correspond to the WP width and its momentum, respectively. $N_{i}=\left(2 \pi \rho_{i}^{2}(t)\right)^{-3 / 4}$ is the normalization factor. The WP function (1) is specified by the timedependent parameters $\left\{q_{\alpha_{i}}(t), p_{\alpha_{i}}(t), \rho_{i}(t), \Lambda_{i}(t)\right\}$ to be determined through the time-dependent variational principle of the action integral. ${ }^{31-33}$ The resulting equations of motions (EOMs) to be solved finally become 


$$
\begin{aligned}
& \dot{q}_{\alpha_{i}}=\frac{p_{\alpha_{i}}}{m_{i}}, \quad \dot{p}_{\alpha_{i}}=-\frac{\partial \sum_{i<j}^{N}\left\langle V_{i j}\left(x_{i j}, y_{i j}, z_{i j}\right)\right\rangle}{\partial q_{\alpha_{i}}}, \\
& \dot{\rho}_{i}=\frac{\Lambda_{i}}{m_{i}}, \quad \dot{\Lambda}_{i}=-\frac{\partial \sum_{i<j}^{N}\left\langle V_{i j}\left(x_{i j}, y_{i j}, z_{i j}\right)\right\rangle}{3 \partial \rho_{i}}+\frac{\hbar^{2}}{4 m_{i} \rho_{i}^{3}}
\end{aligned}
$$

for $i=1,2, \ldots, N$. Here, the intra- and intermolecular potential functions $V_{i j}\left(x_{i j}, y_{i j}, z_{i j}\right)$ are averaged as $\langle X\rangle$ $\equiv\langle\Psi, t|X| \Psi, t\rangle$, and the mass $m_{i}$ and $\hbar$ have been retrieved. Our semiquantum water (SQW) program requires only $2 N$ additional EOM for the auxiliary coordinates and momenta representing the WP widths of hydrogen atoms. The 3D SQW MD simulation was developed by expanding the potentials $V_{i j}\left(x_{i j}, y_{i j}, z_{i j}\right)$ around $\left\langle x_{i}\right\rangle,\left\langle y_{i}\right\rangle$, and $\left\langle z_{i}\right\rangle$ for hydrogen atoms. ${ }^{31}$ The dynamics of oxygen atoms are also influenced by additional nonclassical forces from the semiquantum hydrogen atoms. With the explicit extended potential functions, we can perform the SQW dynamics simulations of 1024 water molecules solving the EOMs (2) and (3). The explicit classical and semiquantum potential functions used in this study are described in our previous paper. ${ }^{31}$ Our classical water potentials correspond to a flexible SPC/Fd water model which can reproduce a reasonable diffusion coefficient. $^{3}$

The SQW and FCW systems are composed of 1024 water molecules and were simulated in a cubic simulation box with a periodic boundary condition. The number of particles and the cubic system volume $V=(31.5)^{3} \AA^{3}$ were kept constant through the simulation. The mass density is set to be $0.982 \mathrm{~g} / \mathrm{cm}^{3}$ which is from the constant pressure Monte Carlo study $^{34}$ on the rigid TIP3P water at $298 \mathrm{~K}, 1 \mathrm{~atm}$. All integration of the EOM is performed by the velocity-verlet method with the time step of 0.1 fs. In the present cooling and equilibration runs, we made only the classical velocity degrees of freedom influenced by the heat baths, the velocity scaling thermostat, and Berendsen methods, with $T=298 \mathrm{~K}$ and the relaxation time of $400 \mathrm{fs}$. Other degrees of freedom are freely time evolved by the EOMs (5) and (6). After the careful cooling and equilibration runs, the whole phase space reaches the thermal equilibrium owing to heat conduction between the degrees of freedom controlled by the heat baths and the other degrees of freedom free from the heat baths. Finally, we carried out the $N V E$ (microcanonical) simulation. The time step of 0.1 fs gives good total energy conservation even for the 1880.69 ps run; the average root-mean-square (rms) deviations of the total energies were 3.13 $\times 10^{-2} \mathrm{kcal} / \mathrm{mol}$ for SQW and $1.76 \times 10^{-2} \mathrm{kcal} / \mathrm{mol}$ for FCW. Another criterion for the trajectory accuracy is the ratio of the rms fluctuation of the total energy over that of the total kinetic energy, for which we obtained $9.26 \times 10^{-4}$ for SQW and $5.4 \times 10^{-4}$ for FCW. The resultant kinetic temperatures were found to be $297.6 \mathrm{~K}$ with the rms fluctuation of $3.69 \mathrm{~K}$ for SQW and $297.4 \mathrm{~K}$ with the rms fluctuation of $3.55 \mathrm{~K}$ for FCW. The results shown in Figs. 1 and 2 were obtained by the microcanonical simulation for the last 380.69 ps during 1880.69 ps. All the other results were from the same microcanonical simulation, but from 100 to 200 ps.

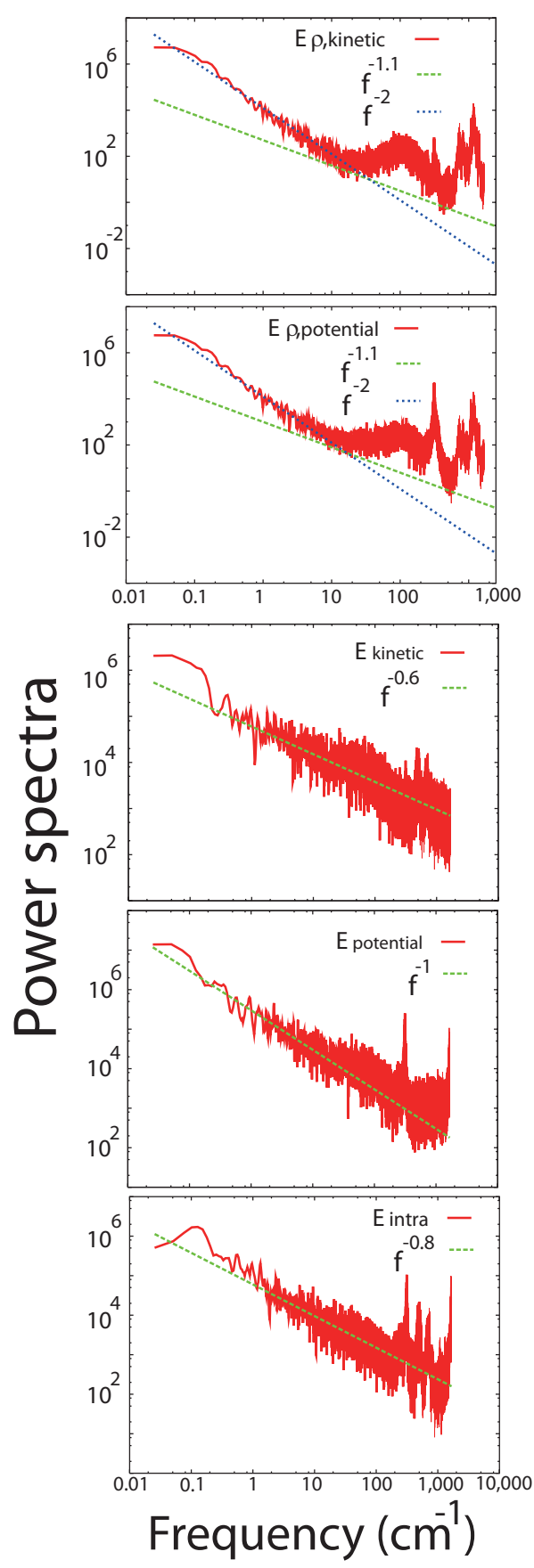

FIG. 1. Power spectra of the kinetic (upper) and potential (upper middle) energies of WPs describing semiquantum hydrogen atoms, and the classical parts of kinetic (middle), potential (lower middle), and intamolecular (lower) energies. The power spectra of the WP kinetic and potential energies show a simple decay $1 / f^{2}$ in the lower frequency region, whereas those of the classical energy parts are all reproduced by the functions $1 / f^{x}$ with the exponents $0.5<x<1.5$. Although the classical energy dynamics follow the well-known multitimescale dynamics, the WP energy dynamics are not strongly coupled to them.

The extra computational costs for SQW over FCW are only marginal, about $20 \%$. Nevertheless, our SQW simulation reproduced the major quantum effects observed in the PIMC, CMD, and ring polymer MD methods; the diffusion constant is increased, the IR peaks are redshifted and the water is less structured. ${ }^{31}$ Our SQW method could be especially effective in biosimulations where a large number of solvating water molecules are often needed to realize stable simulations. 


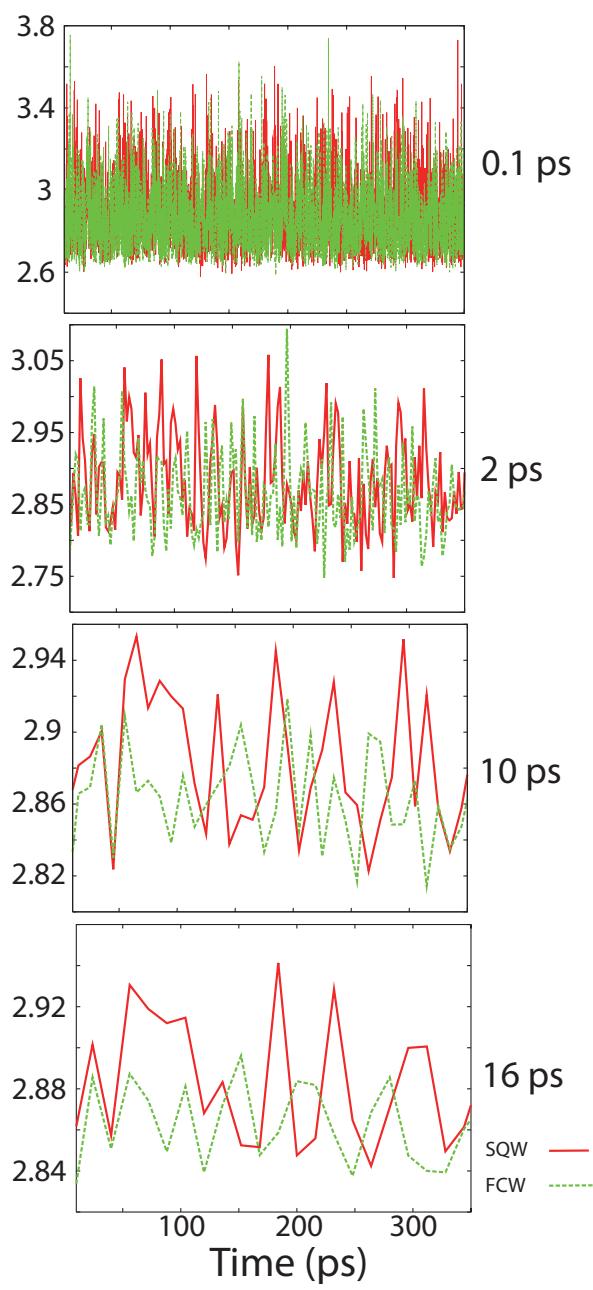

FIG. 2. Averaged dynamics of the maximum peak position of the RDF (in $\AA$ ) around a representative oxygen atom. The time resolutions for averaging are displayed beside each panel. The solid and dashed lines express the semiquantum and classical results, respectively. Descending down the panels, the random thermal fluctuations disappear and the two-state configuration transition appears. The water structure has a strong tendency to have only two configurations corresponding to hydrogen-bond and distorted configurations. The distorted structure for SQW is less structured than that for FCW.

\section{RESULTS AND DISCUSSIONS}

Figure 1 shows power spectra of the kinetic and potential energies of WPs describing the semiquantum hydrogen atoms (the upper two panels). The power spectrum is defined as the absolute square of Fourier transformed energy dynamics, $\left|\int E(t) \exp (i \omega t) d t\right|^{2}$. The power spectra of the lowfrequency region are both well fitted by $1 / f^{2}$, while the higher frequency spectra exhibit the complicated behavior. This is quite different from the classical part of the kinetic, potential, and intramolecular energies in the same SQW simulation, where the entire spectra can be fitted by single functions $1 / f^{x}$ with the exponents $0.5<x<1.5$ (the lower three panels). The power spectra demonstrate that, although the classical parts exhibit multitimescale dynamics as was reported by previous works, ${ }^{30}$ the low-frequency energy dynamics of WPs are rather of single timescale and not strongly influenced by the classical part of the energy fluctuation.

To study the dynamics of the structure, we define the time-dependent radial distribution functions (RDFs) as

$$
g(r, t)=\frac{n(r, t)}{4 \pi r^{2} d r n_{0}},
$$

where $n(r, t)$ represents the number of oxygen atoms between shells of radii $r$ and $r+d r$ from a representative oxygen atom (to be called $i$ th oxygen atom in this paper), and $n_{0}$ is the oxygen number density of the whole system. The RDF dynamics directly reflects the liquid structure rearrangement around the oxygen atom. Figure 2 shows the time-dependent maximum peak positions of the RDFs for pairs of the $i$ th oxygen atom and the other surrounding 1023 oxygen atoms. The panels are different in the time resolution for the averaging as indicated beside each panel. With the finer time resolution, the maximum peak positions for SQW and FCW both fluctuate randomly reflecting the short-time thermal modulations. As the time resolution is coarse grained, the random thermal fluctuations are smoothed and the timedependent maximum peak position exhibits a behavior of two-state transitions between well-structured and lessstructured states. This implies that the water structure has two kinds of configurations corresponding to fully coordinated and distorted local hydrogen-bond configurations. It supports the mixture model which was recently proposed by $\mathrm{x}$-ray experiments and raised a controversy. ${ }^{35-37}$ We also mention that the distorted structure for SQW is less structured than that for FCW, reflecting the smoothed free energy landscape due to the ZPE of the semiquantum hydrogen atoms. $^{31}$

Figure 3 demonstrates that the hydrogen quantum effects most essentially influence the hydrogen-bond dynamics. We calculated the number of water molecules hydrogen bond to the $i$ th water molecule as a function of time, $N_{\mathrm{HB}}(t)$. The common geometrical criterion for hydrogen bonds was used: $^{3}$ i.e., if the distance between two oxygen atoms, $r_{\mathrm{OO}}(t)$, is less than $3.5 \AA$ and the $\mathrm{OH} \cdots \mathrm{O}$ angle is greater than $150^{\circ}$, the water pair is considered as hydrogen bonded. The evolution of $N_{\mathrm{HB}}(t)$ directly reflects the HBN dynamics. ${ }^{1}$ The upper panel in Fig. 3 shows the normalized time correlation functions (TCFs) $\left\langle N_{\mathrm{HB}}(t) N_{\mathrm{HB}}(0)\right\rangle /\left\langle N_{\mathrm{HB}}^{2}(0)\right\rangle$. The TCF for SQW (solid) decreases faster than that for FCW (dashed), indicating that the quantum effect of hydrogen atoms accelerates the memory decay of the HBN. This tendency seems in accord with the experimentally observed ultrafast memory loss in liquid water, ${ }^{38}$ with an assumption that the HBN structure is closely related to the $\mathrm{OH}$ stretching modes. In addition, the shoulder in the decay curve for FCW in the early stage $(\sim 10 \mathrm{fs})$ disappears for the SCW case, suggesting that the rearrangement of the $\mathrm{HBN}$ is made smoother due to the quantum effects. The middle and lower panels are the power spectra of the HBNs, $\left|\int N_{\mathrm{HB}}(t) \exp (i \omega t) d t\right|^{2}$, for SQW and FCW, respectively. As seen, a notable qualitative difference is found: the hydrogen-bond dynamics of the quantized water exhibit an almost complete $1 / f$ spectrum especially in the low-frequency region that reflects multitimescale dynamics, whereas in the FCW case the exponents of the power spectrum are obviously out of the multitimescale region, $0.5<x<1.5$.

To further demonstrate the significance of the quantum dynamics, we show in Fig. 4(a) the time evolutions of the 

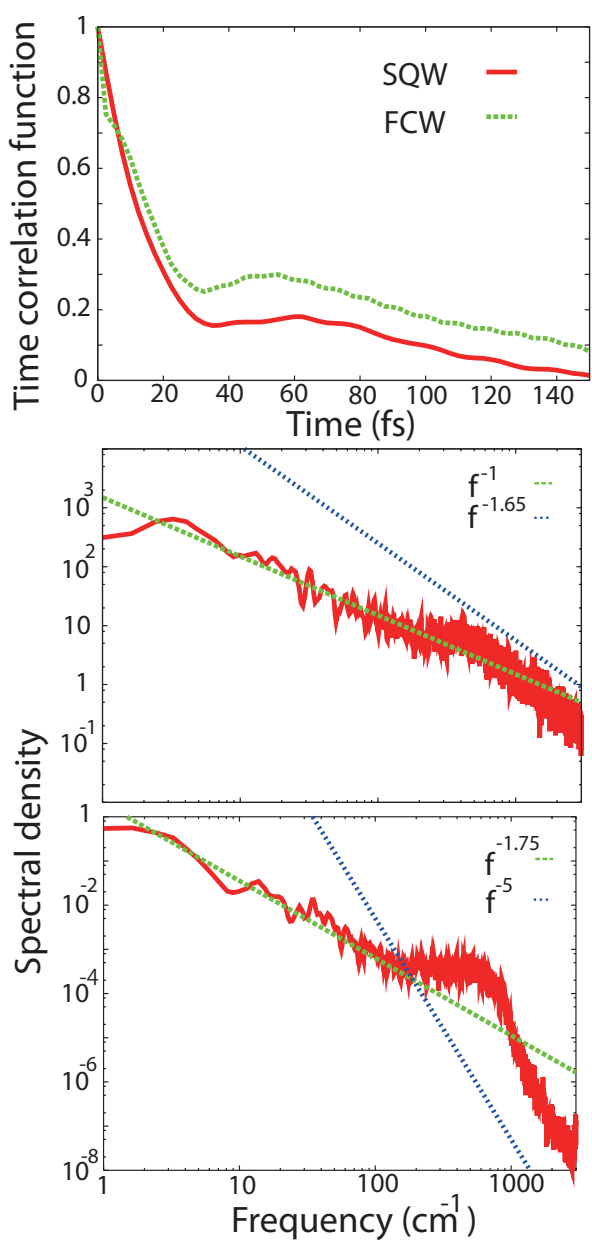

FIG. 3. Dynamics of the number of hydrogen bonds $N_{\mathrm{HB}}(t)$ around a representative water molecule. The upper figure shows the normalized TCFs of $N_{\mathrm{HB}}(t)$ for SQW (solid line) and FCW (dashed line). The faster decay in SQW demonstrates the more rapid memory loss in the HBN due to the quantum hydrogen atoms. The middle and lower figures show the power spectra of $N_{\mathrm{HB}}(t)$ for SQW and FCW, respectively. The complete $1 / f$ spectra in SQW indicate the multitimescale hydrogen-bond dynamics, which contrasts with the FCW case where the dynamics are rather of single timescale.

WP width of the hydrogen atom $\rho_{i}(t)$, the average $\mathrm{OH}$ bond length $\left(r_{i, \mathrm{OH}_{1}}(t)+r_{i, \mathrm{OH}_{2}}(t)\right) / 2$ of the $i$ th water molecule, and the number of hydrogen bonds $N_{\mathrm{HB}}(t)$ to the $i$ th water molecule. All the data were time averaged over every $1.25 \mathrm{ps}$. As indicated by the circles, notable correlations among these three variables are observed. When the WP width increases, both the $\mathrm{OH}$ bond length and the hydrogen-bond number decrease. Although these correlations are not complete, reflecting complicated fluctuations with coupling to other variables, they are seen not only in the shown timescale in Fig. 4(a) but also throughout our SQW MD simulations. The significant correlations are observed particularly in the characteristic regions where the dynamics turn over, as marked by the circles. As the WP width grows, the $\mathrm{OH}$ bond length becomes small due to the excluded volume effect and repulsive force induced by the WP semiquantum hydrogen atoms. The decrease in the $\mathrm{OH}$ bond length leads to less structured liquid and results in weakening the HBN. In other words, the quantum WP delocalizations suppress the hydrogen-bond structure and result in the higher mobility of waters out of the HBN.

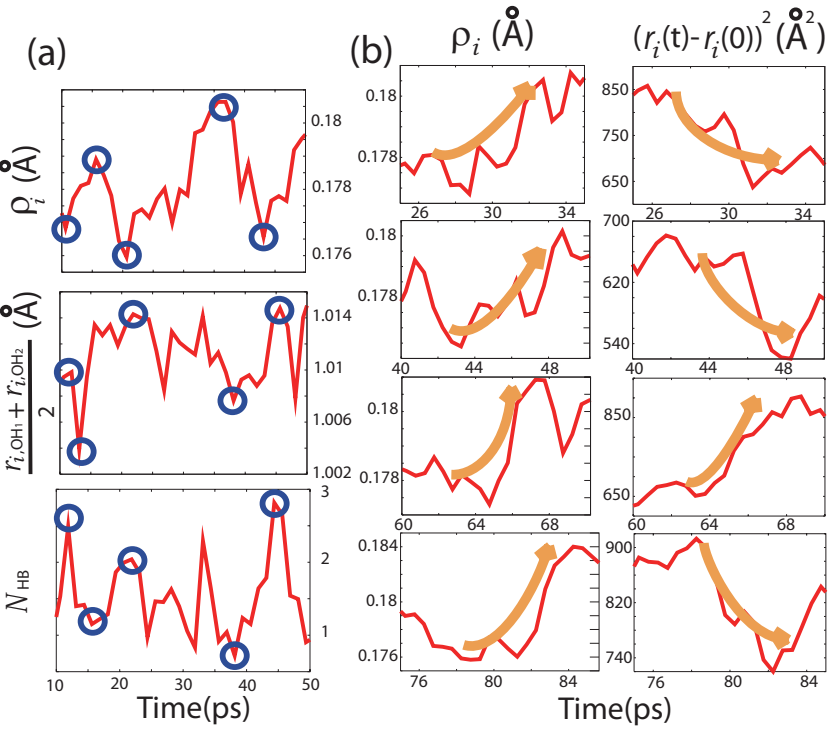

FIG. 4. WP dynamics accompanying the water rearrangement dynamics. (a) Dynamics of the WP width (upper), the average $\mathrm{OH}$ bond length (middle), and the number of hydrogen bonds (lower) for a representative water molecule. The circles mark notable correlations among these three variables in the characteristic regions where their dynamics turn over. (b) Dynamics of the WP width (left column) and the mean-square displacement of the oxygen atom (right column) for a representative water molecule. The arrows indicate that the significant changes in the mean-square displacement are always accompanied by the WP broadening.

Figure 4(b) displays the WP dynamics (left column) and the corresponding mean-square displacements (right column) of the $i$ th oxygen atom, $\left|\mathbf{r}_{i}(t)-\mathbf{r}_{i}(0)\right|^{2}$, where $\mathbf{r}_{i}(t)$ denotes the position vector of the $i$ th oxygen atom at time $t$. The arrows in the right column mark significant changes in the meansquare displacement which are considered to represent the motion of the $i$ th molecule such as a cage breakout. These large displacements are always accompanied by the WP broadening, as the arrows in the left column indicate. This correlation is observed for both the increase and decrease in the $\mathbf{r}_{i}(t)$ displacements. The WP broadening/shrinking motion is significantly correlated with the extent of hydrogen bonding around the oxygen atom, and the water configuration changes simultaneously. Although these correlations are not complete due to the complicated fluctuations, there are actually significant correlations between the left and corresponding right figures in Fig. 4(b). This provides a physically intuitive picture for the increased diffusion constant and the reduced structure reported in the SQW simulations. ${ }^{16,17,23,31}$

\section{CONCLUDING REMARKS}

We for the first time directly viewed the quantum dynamics of hydrogen atoms in bulk liquid water. We found the significant correlations between the water hydrogen WP dynamics and the HBN dynamics and demonstrated their actual influences on the HBN rearrangement. The results provide new physical insights on the HBN dynamics in water which are important not only for describing the pure liquid water dynamics but also for understanding chemical reactions and biomolecule dynamics in water. 


\section{ACKNOWLEDGMENTS}

K.H.D. is partially supported by Grant-in-Aids for Scientific Research from Japan Society for the Promotion of Science (KAKENHI), Grant No. 20750010, and the grant from the Kyoto University Foundation. K.A. acknowledges support from KAKENHI in priority areas "Molecular Theory for Real Systems" (Grant No. 190296) and "Emergency of Highly Elaborated $\pi$-space and its Function" (Grant No. 20106017).

${ }^{1}$ D. Laage and J. T. Hynes, Science 311, 832 (2006).

${ }^{2}$ L. X. Dang and B. M. Pettitt, J. Phys. Chem. 91, 3349 (1987).

${ }^{3}$ Y. Wu, H. L. Tepper, and G. A. Voth, J. Chem. Phys. 124, 024503 (2006).

${ }^{4}$ D. M. Ferguson, J. Comput. Chem. 16, 501 (1995).

${ }^{5}$ I. G. Tironi, R. M. Brunne, and W. F. vanGunsteren, Chem. Phys. Lett. 250, 19 (1996).

${ }^{6}$ T. Yagasaki and S. Saito, J. Chem. Phys. 128, 154521 (2008).

${ }^{7}$ K. Toukan and A. Rahman, Phys. Rev. B 31, 2643 (1985).

${ }^{8}$ S. Iuchi, A. Morita, and S. Kato, J. Phys. Chem. B 106, 3466 (2002).

${ }^{9}$ I. Ohmine and S. Saito, Acc. Chem. Res. 32, 741 (1999).

${ }^{10}$ H. J. Bakker and H. K. Nienhuys, Science 297, 587 (2002).

${ }^{11}$ J. M. Headrick, E. G. Diken, R. S. Walters, N. I. Hammer, R. A. Christie, J. Cui, E. M. Myshakin, M. A. Duncan, M. A. Johnson, and K. D. Jordan, Science 308, 1765 (2005).

${ }^{12}$ J. A. Morrone and R. Car, Phys. Rev. Lett. 101, 017801 (2008).

${ }^{13}$ L. H. de la Pena, M. S. G. Razul, and P. G. Kusalik, J. Phys. Chem. A 109, 7236 (2005).

${ }^{14}$ E. G. Noya, C. Vega, L. M. Sese, and R. Ramirez, J. Chem. Phys. 131, 124518 (2009).

${ }^{15}$ B. S. Gonzalez, E. G. Noya, C. Vega, L. M. Sese, and R. Ramirez, J. Phys. Chem. B 114, 2484 (2010).

${ }^{16}$ R. A. Kuharski and P. J. Rossky, J. Chem. Phys. 82, 5164 (1985).

${ }^{17}$ A. Wallqvist and B. J. Berne, Chem. Phys. Lett. 117, 214 (1985).

${ }^{18}$ J. A. Poulsen, G. Nyman, and P. J. Rossky, Proc. Natl. Acad. Sci. U.S.A.
102, 6709 (2005)

${ }^{19}$ F. Paesani, S. S. Xantheas, and G. A. Voth, J. Phys. Chem. B 113, 13118 (2009).

${ }^{20}$ S. Habershon, T. E. Markland, and D. E. Manolopoulosa, J. Chem. Phys. 131, 024501 (2009).

${ }^{21}$ S. Habershon, G. S. Fanourgakis, and D. E. Manolopoulosb, J. Chem. Phys. 129, 074501 (2008).

${ }^{22}$ T. F. Miller and D. E. Manolopoulos, J. Chem. Phys. 123, 154504 (2005).

${ }^{23}$ F. Paesani, W. Zhang, D. A. Case, T. E. Cheatham, and G. A. Voth, J. Chem. Phys. 125, 184507 (2006).

${ }^{24}$ G. S. Fanourgakis, G. K. Schenter, and S. S. Xantheasa, J. Chem. Phys. 125, 141102 (2006).

${ }^{25}$ S. Habershon and D. E. Manolopoulos, J. Chem. Phys. 131, 244518 (2009).

${ }^{26}$ J. Liu, W. H. Miller, F. Paesani, W. Zhang, and D. A. Case, J. Chem. Phys. 131, 164509 (2009).

${ }^{27}$ L. H. de la Pena and P. G. Kusalik, J. Am. Chem. Soc. 127, 5246 (2005).

${ }^{28}$ F. Paesani, S. Iuchi, and G. A. Voth, J. Chem. Phys. 127, 074506 (2007).

${ }^{29}$ F. Paesani and G. A. Voth, J. Phys. Chem. B 113, 5702 (2009).

${ }^{30}$ M. Sasai, I. Ohmine, and R. Ramaswamy, J. Chem. Phys. 96, 3045 (1992).

${ }^{31}$ K. Hyeon-Deuk and K. Ando, J. Chem. Phys. 131, 064501 (2009).

${ }^{32}$ K. Ando, J. Chem. Phys. 121, 7136 (2004).

${ }^{33}$ K. Ando, J. Chem. Phys. 125, 014104 (2006).

${ }^{34}$ W. L. Jorgensen, J. Chandrasekhar, J. D. Madura, R. W. Impey, and M. L. Klein, J. Chem. Phys. 79, 926 (1983).

${ }^{35}$ T. Tokushima, Y. Harada, O. Takahashi, Y. Senba, H. Ohashi, L. G. M. Pettersson, A. Nilsson, and S. Shin, Chem. Phys. Lett. 460, 387 (2008).

${ }^{36} \mathrm{Ph}$. Wernet, D. Nordlund, U. Bergmann, M. Cavalleri, M. Odelius, H. Ogasawara, L. A. Näslund, T. K. Hirsch, L. Ojamäe, P. Glatzel, L. G. M. Pettersson, and A. Nilsson, Science 304, 995 (2004).

${ }^{37}$ J. D. Smith, C. D. Cappa, K. R. Wilson, B. M. Messer, R. C. Cohen, and R. J. Saykally, Science 306, 851 (2004).

${ }^{38}$ M. L. Cowan, B. D. Bruner, N. Huse, J. R. Dwyer, B. Chugh, E. T. J. Nibbering, T. Elsaesser, and R. J. D. Miller, Nature (London) 434, 199 (2005). 